\title{
Rare paradoxical adverse event in Crohn's disease: a case report
}

\author{
Marina Okoshi ${ }^{1}$, Hiroki Sato ${ }^{2}$, Terasu Honma ${ }^{1}$, Shuji Terai ${ }^{2}$ \\ ${ }^{1}$ Division of Gastroenterology and Hepatology, Saiseikai Niigata Daini Hospital, Niigata, Japan; ${ }^{2}$ Division of Gastroenterology and Hepatology, \\ Niigata University Medical and Dental Hospital, Niigata, Japan \\ Correspondence to: Hiroki Sato, MD, PhD. Division of Gastroenterology, Niigata University Medical and Dental Hospital, 757-1, Asahimachidori, \\ Chuo-ku, Niigata-city, Niigata 951-8510, Japan. Email: pyloki-sato@med.niigata-u.ac.jp.
}

\begin{abstract}
An 18-year-old male adolescent diagnosed with ileocolitis-type Crohn's disease received therapy involving an anti-tumor necrosis factor- $\alpha(\mathrm{TNF}-\alpha)$ blocker, which resulted in remission for 2 years. The patient presented with fever, stomatitis, and cough with respiratory distress. Chest radiography revealed bilateral hilar lymphadenopathy; bronchoalveolar lavage showed high lymphocyte level: 40.8\% and CD4/8 value: 3.9. Transbronchial lung biopsy identified multiple non-caseating granulomas fused to each other with connective tissue septa, leading to the diagnosis of sarcoidosis. The sarcoidosis was defined as a paradoxical adverse event (PAE) due to the use of TNF- $\alpha$ blocker; therefore, cessation of TNF- $\alpha$ blocker administration was sufficient to induce remission. We report a rare case of PAE on Crohn's disease. The characteristics and distinguishing histologies of PAE and Crohn's disease are described. Biological agent therapy has been reported to cause several PAEs during the treatment of immune-mediated inflammatory disorders. PAEs are defined as pathological conditions that occur during biological agent therapy usually effective in treatment. The laboratory, radiography, and histological findings shown in this case are useful for differential diagnosis and management of PAE.
\end{abstract}

Keywords: Anti-tumor necrosis factor- $\alpha(\mathrm{TNF}-\alpha)$ blocker; Crohn's disease; sarcoidosis; paradoxical adverse event (PAE); case report

Submitted Jul 17, 2019. Accepted for publication Dec 11, 2019.

doi: 10.21037/atm.2019.12.122

View this article at: http://dx.doi.org/10.21037/atm.2019.12.122

\section{Introduction}

Biological agent therapy has been reported to cause several paradoxical adverse events (PAEs) during treatment of immune-mediated inflammatory disorders. PAEs are defined as pathological conditions during biological agent therapy that is usually effective in treatment (1). Herein, we report a rare case of PAEs occurring in a patient with Crohn's disease.

\section{Case presentation}

An 18-year-old male adolescent was referred to our hospital for diarrhea, fever (temperature $>38^{\circ} \mathrm{C}$ ), stomatitis, and perianal abscess for 4 months. He had no allergies, no significant medical history, and was not on any medication. Colonoscopy revealed multiple erosions in the terminal ileum and extensive longitudinal ulcers in the colon (Figure 1). Therefore, several biopsies were obtained that showed poorly formed non-caseating granulomas without a well-demarcated septum (Figure 1 insert). He was diagnosed with ileocolitis-type Crohn's disease. A combination of an elemental diet and oral prednisolone (starting dose of $0.6 \mathrm{mg} / \mathrm{kg} /$ day, subsequently tapered) resulted in remission, but the disease relapsed because of poor patient compliance. Furthermore, he received therapy involving an anti-tumor necrosis factor- $\alpha(\mathrm{TNF}-\alpha)$ blocker (infliximab, $5 \mathrm{mg} / \mathrm{kg}$ during weeks 0,2 , and 6 ; subsequently, once every 8 weeks), which resulted in remission for 2 years. However, after the remission, he was lost to follow-up.

Eleven months later, he presented with fever, stomatitis, and cough with respiratory distress that had lasted for 3 months. His peripheral blood white blood cell count and C-reactive protein level were slightly elevated at 


\section{Page 2 of 3}

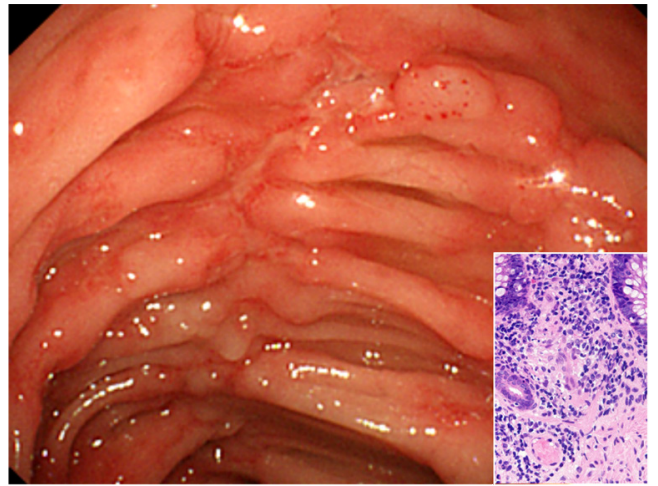

Figure 1 Colonoscopy revealed multiple erosions in the terminal ileum and extensive longitudinal ulcers in the colon. Insert: histology showed poorly formed non-caseating granulomas without a well-demarcated septum, leading to the diagnosis of Crohn's disease (hematoxylin-eosin staining, 100× magnification).

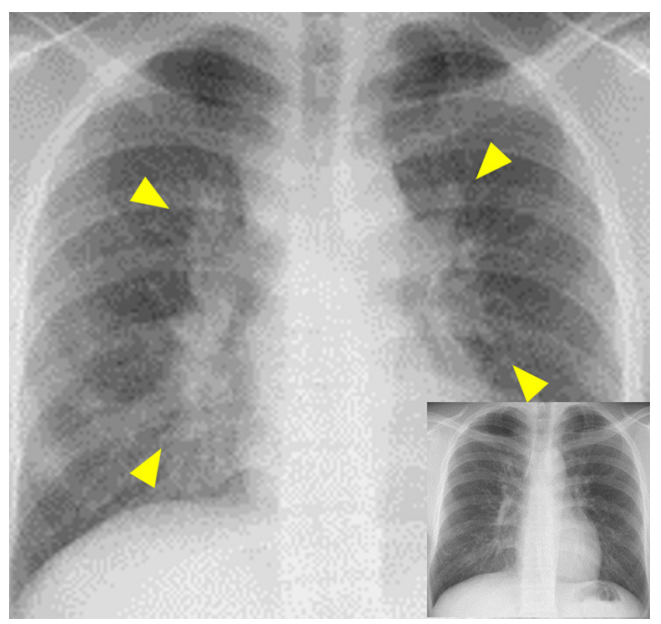

Figure 2 Chest radiography revealed bilateral hilar lymphadenopathy (yellow triangle), which was naturally resolved after 8 months (insert).

10,100/ $\mathrm{LL}$ (neutrophils: $72.5 \%$ ) and $2.29 \mathrm{mg} / \mathrm{mL}$ (reference: $<0.20$ ), respectively. Several tests inspecting infection as tuberculosis and sarcoidosis were negative. Additionally, serum TNF- $\alpha$ level was abnormally elevated at $117.4 \mathrm{pg} / \mathrm{mL}$ (reference: $<2.8$ ). The serum angiotensinconverting enzyme (ACE), soluble interleukin-2 receptor (sIL$2 \mathrm{R}$ ), and lysozyme levels were elevated at $70.2 \mathrm{U} / \mathrm{L}$ (reference range, 7.0-25.0 U/L), 10,048 U/mL (122-496 U/L), and $35.8 \mu \mathrm{g} / \mathrm{mL}(5.0-10.0 \mu \mathrm{g} / \mathrm{mL})$, respectively. Furthermore, a chest radiograph revealed bilateral hilar lymphadenopathy

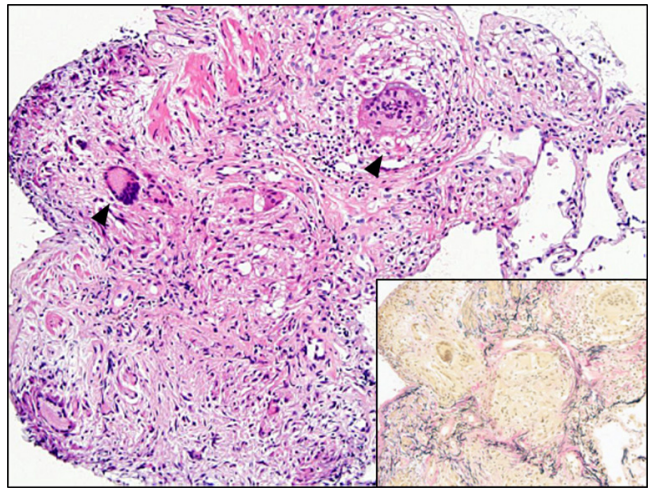

Figure 3 Transbronchial lung biopsy identified multiple noncaseating granulomas fused to each other with connective tissue septa, leading to a diagnosis of sarcoidosis (black triangles, giant cells) (hematoxylin-eosin staining, $100 \times$ magnification). Insert: Elastica van Gieson staining (100× magnification).

(Figure 2, yellow triangle), and bronchoalveolar lavage showed a high lymphocyte level: $40.8 \%$ (reference $<15 \%$ ) and CD4/8 value: 3.9 (reference $<3.5$ ). Transbronchial lung biopsy identified multiple non-caseating granulomas fused to each other with connective tissue septa, leading to a diagnosis of sarcoidosis (Figure 3, black triangles, giant cells; insert: Elastica van Gieson staining).

Cessation of TNF- $\alpha$ blocker administration was sufficient to induce remission.

\section{Discussion}

Cases of sarcoidosis and granulomatosis-like diseases that occur during or after treatment with immune checkpoint inhibitors, highly active antiretroviral therapy, interferons, and TNF- $\alpha$ blocker have been reported (2), although cases of Crohn's disease are still scarce. The mechanism of the reaction remains unclear, however, in our case, the paradoxical increase in TNF- $\alpha$ activity may have triggered the disease because TMF- $\alpha$ plays an important role in the formation and maintenance of sarcoid granuloma (3). The prognosis of sarcoidosis as a PAE seems favorable, and in most cases, cessation of TNF- $\alpha$ blocker administration is sufficient to induce remission (Figure 2 insert, 8 months later). In cases treated with biological agents, including TNF- $\alpha$ blocker, a careful follow-up and differential diagnoses of PAEs are necessary even after treatment cessation. For the diagnosis of sarcoidosis, there are no specific or sensitive symptoms or physical findings. 
However, the specificity and sensitivity of elevated serum ACE and sIL-2R level, respectively, were reported to be high $(4,5)$, which are useful for differential diagnosis. Furthermore, bilateral hilar lymphadenopathy on chest radiography is also a characteristic finding. Thereafter, non-caseating granulomas on histology should be proved, and if the disease is in the lungs, elevated lymphocyte on bronchoalveolar lavage is also supplementary (6). We should consider lung infections or metastatic Crohn's disease (7), which have more severe pathology when compared with our case. Only case report and case series are present in literature, which can be considered a limitation, however, our case suggests that laboratory, radiography, and histological findings are useful for the differentiation and management of sarcoidosis as PAE.

\section{Acknowledgments}

None.

\section{Footnote}

Conflicts of Interest: The authors have no conflicts of interest to declare.

Ethical Statement: The authors are accountable for all aspects of the work in ensuring that questions related to the accuracy or integrity of any part of the work are appropriately investigated and resolved. Written informed consent was obtained from the patient for publication of this manuscript and any accompanying images.

Cite this article as: Okoshi M, Sato H, Honma T, Terai S. Rare paradoxical adverse event in Crohn's disease: a case report. Ann Transl Med 2020;8(4):133. doi: 10.21037/atm.2019.12.122

\section{References}

1. Toussirot É, Aubin F. Paradoxical reactions under TNFalpha blocking agents and other biological agents given for chronic immune-mediated diseases: an analytical and comprehensive overview. RMD Open 2016;2:e000239.

2 Chopra A, Nautiyal A, Kalkanis A, Judson MA. Drug-Induced Sarcoidosis-Like Reactions. Chest 2018;154:664-77.

3 Marzouk K, Saleh S, Kannass M, et al. Interferon-induced granulomatous lung disease. Curr Opin Pulm Med 2004;10:435-40.

4 Ungprasert P, Carmona EM, Crowson CS, et al. Diagnostic Utility of Angiotensin-Converting Enzyme in Sarcoidosis: A Population-Based Study. Lung 2016;194:91-5.

5 Thi Hong Nguyen C, Kambe N, et al. Serum soluble interleukin-2 receptor level is more sensitive than angiotensin-converting enzyme or lysozyme for diagnosis of sarcoidosis and may be a marker of multiple organ involvement. J Dermatol 2017;44:789-97.

6 Statement on sarcoidosis. Joint Statement of the American Thoracic Society (ATS), the European Respiratory Society (ERS) and the World Association of Sarcoidosis and Other Granulomatous Disorders (WASOG) adopted by the ATS Board of Directors and by the ERS Executive Committee, February 1999. Am J Respir Crit Care Med 1999;160:736-55.

7 Gill KR, Mahadevan U. Infliximab for the treatment of metastatic hepatic and pulmonary Crohn's disease. Inflamm Bowel Dis 2005;11:210-2. 